\title{
Dysphasia Accompanied by Periodic Lateralized Epileptiform Discharges
}

\author{
Shin-ichi Ono, Koichi ChIDA, Nakahide FukayA*, Hirokazu Yoshinashi** and Toshiaki Takasu**
}

\begin{abstract}
We encountered a 67-year-old man who presented with repetitious dysphasia accompanied by periodic lateralized epileptiform discharges (PLEDs) on the electroencephalogram. A good correlation was established between the dysphasia and the PLEDs. A persistent partial seizure accompanied by PLEDs originating in the left hemisphere presented with dysphasia clinically. None of the previously reported patients with epileptic dysphasia had accompanying PLEDs. The administration of carbamazepine was successful to terminate the seizure, however valproate was not. This case indicates that PLEDs may produce significant, however reversible, functional damage, and may advance our understanding of the pathophysiology of epileptic dysphasia. (Internal Medicine 36: 59-61, 1997)
\end{abstract}

Key words: periodic lateralized epileptiform discharges (PLEDs), partial seizure, epileptic dysphasia

\section{Introduction}

A brief period of impaired consciousness and/or motor paresis sometimes follows a convulsion (1). This transient period has been recognized as a postictal state, during which some patients cannot express words spontaneously or comprehend what other people say and exhibit motor paresis (1). Apart from the postictal state, epilepsy itself on rare occasions may cause speech disturbance: epileptic dys- or aphasia (2). However, if dysphasia is the sole clinical manifestation, it is very difficult to differentiate between the above two conditions. The diagnosis of epileptic dysphasia is especially difficult, because of the difficulty of identifying a clear correlation between dysphasia and electrical seizure on the electroencephalogram (EEG).

Periodic lateralized epileptiform discharges (PLEDs) is a relatively uncommon EEG phenomenon consisting of periodic or quasi-periodic spikes or sharp waves, occurring at 1-2 second intervals in association with structural brain lesions (3). We encountered a patient with epileptic dysphasia accompanied by PLEDs on the EEG. We report the patient and describe a clear relationship between the dysphasia and PLEDs.

\section{Case Report}

A 67-year-old right handed man was admitted to the emergency ward of our hospital on December 4, 1991 because of a generalized tonic-clonic convulsion. The convulsion was successfully terminated by intravenous administration of anticonvulsants, but mild dysphasia persisted. EEGs obtained on December 5 and 10, when the patient had dysphasia, exhibited PLEDs in the left hemisphere. The PLEDs persisted throughout the recording time, about 30 minutes. The dysphasia gradually faded away over a few weeks. The patient was treated with sodium valproate and discharged on December 30. On January 31, 1992 he was admitted to a local hospital because of a recurrence of the convulsions. After the convulsions stopped, the patient again experienced dysphasia for a few weeks, and another EEG on that day showed PLEDs on the same side throughout the recording time. The dosage of sodium valproate was increased because the blood concentration of the drug was below the therapeutic range, and the patient was transferred to our hospital on February 17 for further examination concerning a possible relationship between the dysphasia and the convulsions.

The patient is a retired truck driver. He had a 10-year-history of hypertension well-controlled by medication. He had no family history of convulsive diseases or past history of convulsions, and no diabetes mellitus, drug abuse, history of cerebrovascular accidents or cardiac disease. He consumed large amounts of alcoholic beverages and smoked a pack of cigarettes a day for many years, but stopped virtually all drinking of alcohol and smoking when his hypertension was diagnosed. On physical examination, his blood pressure was $150 / 90 \mathrm{mmHg}$,

From the Department of Neurology, Nihon University Surugadai Hospital, *Fukaya Clinic and **the Department of Neurology, Nihon University Itabashi Hospital, Tokyo

Received for publication March 19, 1996; Accepted for publication November 18, 1996

Reprint requests should be addressed to Dr. Shin-ichi Ono, the Department of Pathology, Health Sciences Centre, University of Western Ontario, London, Ontario N6A 5C1, Canada 
pulse 92 , and temperature $36.2^{\circ} \mathrm{C}$. The heart and abdomen were normal except for a small healed surgical scar in the right lower quadrant. There was no peripheral edema. On neurological examination, the patient was alert and responded to the examiner, except for disorientation to place. His speech was slightly nonfluent. He had minimal perseveration. Cranial nerves and muscle strength were normal. Deep tendon reflexes were normal and equal, but plantar responses were extensor bilaterally. His gait was slightly ataxic and the finger-to-nose test showed mild dysmetria. Sensation was normal for all modalities. The results of the following laboratory tests were normal: urinalysis, complete blood count, liver and renal function and electrolytes. The blood level of sodium valproate was within the therapeutic range. An EEG on February 20, when he no longer had speech disturbance, yielded no epileptic discharges, but showed almost continuous irregular slow waves at the left posterior quadrant (Fig. 1). No visual symptoms such as hemianopsia were observed as mentioned previously. Magnetic resonance imaging of the brain revealed numerous punctate small vascular lesions (i.e. lacunar infarctions), and mild brain atrophy with slight dilatation of the ventricular system.

On March 8, another mild generalized convulsion occurred and was again followed by dysphasia. No initial focal symptoms were noticed before the convulsion. The patient was alert and conscious, but did not comprehend well what the examiner said, since he could not respond appropriately even to simple verbal commands. He was unable to repeat the words that the examiner said. His spontaneous speech was greatly reduced and he articulated words with great effort. He sometimes stammered words out. Naming difficulty and paraphasia were characteristic. An EEG on March 9 again showed PLEDs on the left side (Fig. 2), persisting throughout the recording time. A neurological examination on March 10 disclosed that he could not read or write even short, simple words, and he could not perform easy calculations. He had slight right hemiplegia, presumably Todd's paralysis, but could feed himself appropriately with chopsticks. On March 18, another neurological examination revealed severe perseveration and questionable asomatognosia, but there was no right-left disorientation. No automatism, emotional or mental changes were observed throughout his course. These symptoms disappeared gradually over a few weeks. Sodium valproate was substituted for carbamazepine, and thereafter the patient never had a convulsion or dysphasia during a follow-up period of 3 years, except for one minor seizure without dysphasia. A follow-up EEG obtained on May 1, 1992, when the patient was free of speech disturbance, showed no epileptic discharges.

\section{Discussion}

This case was characterized by repetitive generalized convulsions, each of which was followed by dysphasia accompanied by persistent PLEDs. It is well known that brief periods of speech disturbance follow seizures because of postictal paralysis involving both the speech and motor areas (1). Apart from this condition, a seizure itself may cause speech disturbance:

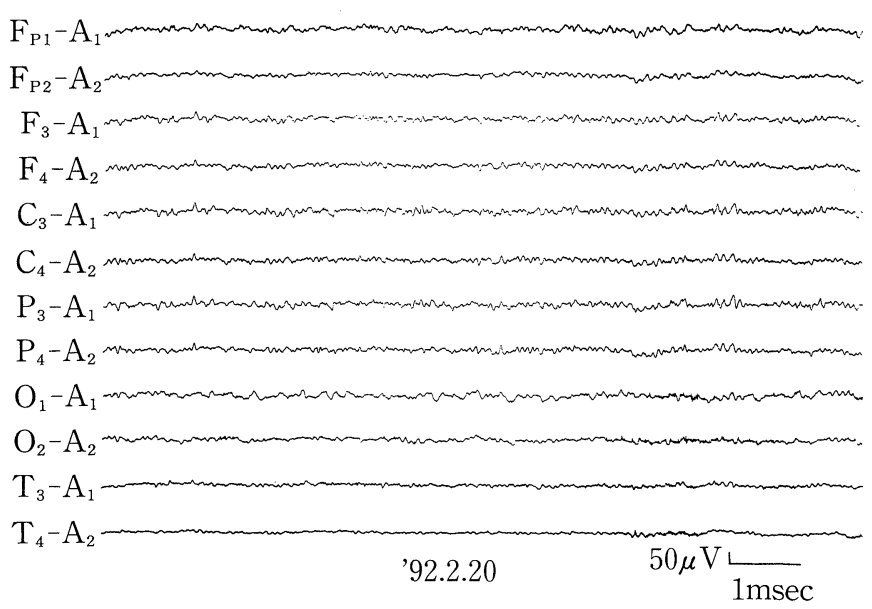

Figure 1. An electroencephalogram recorded on Feb. 20, 1992 when the patient was free of speech disturbance, shows no periodic lateralized epileptiform discharges, but almost continuous irregular slow waves at the left posterior quadrant.

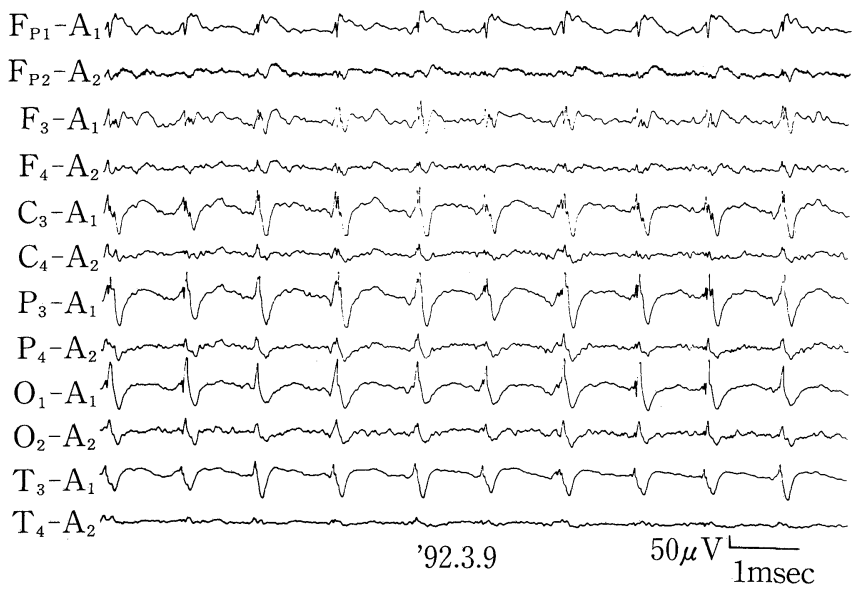

Figure 2. An electroencephalogram recorded on Mar.9, 1992 when the patient had marked dysphasia, reveals periodic lateralized epileptiform discharges in the left hemisphere.

epileptic dysphasia (2). Partial seizure presenting with dysphasia as the sole clinical manifestation, in particular, should be carefully differentiated from cerebrovascular accidents, especially transient ischemic attack (TIA), or psychiatric problems as well as postictal speech disturbance. From the therapeutic point of view, it is very important to differentiate epileptic dysphasia from TIA or psychiatric problems, since the former requires an appropriate antiepileptic agent. Dysphasia may be observed in complex partial seizures (1). A partial seizure with an altered consciousness level is defined as a complex partial seizure, which may overshadow speech disturbance (4). This makes a diagnosis of epileptic dysphasia much more difficult. Associated manifestations, such as automatism, emotional and mental changes, or special sensory symptoms, may strongly 


\section{Dysphasia and PLEDs}

suggest complex partial seizures (1). A good correlation between the electrical seizures on EEG and the clinical symptoms should be required to establish an adequate diagnosis of epileptic dysphasia.

We diagnosed the present patient as having epileptic dysphasia for the following reasons: 1) PLEDs were recorded only in association with periods of dysphasia with the best reproducibility.2) The resolution of dysphasia with the administration of anticonvulsant in the absence of the administration of antiplatelet agent strongly speaks against TIA, although patients with acute cerebrovascular lesions and watershed-type infarctions often exhibit prolonged focal motor attacks that are usually associated with PLEDs (3).

We encountered a patient with partial seizures originating in the dominant hemisphere, which resulted in dysphasia. The generalized convulsions may have been followed by the partial seizures, or may have been secondary generalization of other clinically unrecognized and missed minor seizures. The periods of dysphasia were unusually long for an epileptic dysphasia, since speech disturbance in epileptic dysphasia is quick, fleeting, and faint in nature (5). The long duration of the dysphasia, approximately a few weeks, was compatible with status epilepticus, and PLEDs have been recognized to represent status epilepticus on electroencephalograph (6).

Epileptic dysphasia is a rare clinical condition (7-19), and the present case is noteworthy because of the prolonged dysphasia with persistent PLEDs which persisted throughout the recording time. Although Wells CR (19) reported a case of epileptic dysphasia and PLEDs appeared transiently in that case, none of the previous cases of epileptic dysphasia were accompanied by persistent PLEDs as the present case. We revealed that PLEDs produced reversible significant functional damages as much as, or more than ictal event did, and valproate was not at all the first-choice for our patient with partial seizures. In view of the high correlation between cerebrovascular disease and PLEDs $(3,6)$, a probable underlying pathophysiologic cause may be cerebrovascular in the present case. The results of magnetic resonance imaging of the brain support this probability. We believe that there is no possibility of a connection with alcohol withdrawal, since he stopped drinking alcoholic beverages 10 years before the onset. We also believe that the patient's history of heavy alcohol drinking played a signifi- cant role in the brain atrophy and mild ataxia.

\section{References}

1) Goldensohn ES, Glaser GH, Goldberg MA. Epilepsy. in: Merritt's Textbook of Neurology, 8th ed., Rowland LP, Ed. Lea \& Febiger, Philadelphia, London, 1989, p.780.

2) Alajouanine T, Sabouraud O. Paroxysmal disorders of speech in epilepsy. (Clinical study). Encephale 49: 95, 1960 (in French).

3) Chatrian GE, Shaw C-M, Leffman H. The significance of periodic lateralized epileptiform discharges in EEG. An electrographic, clinical and pathological study. Electroencepharogr Clin Neurophysiol 17: 177, 1964.

4) Gabr M, Luders H, Dinner D, Morris H, Wyllie E. Speech manifestations in lateralization of temporal lobe seizures. Ann Neurol 25: 82, 1989.

5) Cascino GD, Westmoreland BF, Swanson TH, Sharbrough FW. Seizureassociated speech arrest in elderly patients. Mayo Clin Proc 66: 254, 1991.

6) Snodgrass SM, Tsuburaya K, Ajmone-Marsan C. Clinical significance of periodic lateralized epileptiform discharges. Relationship with status epilepticus. J Clin Neurophysiol 6: 159, 1989.

7) De Pasquet EG, Gaudin ES, Bianchi A, De Mandilaharsu SA. Prolonged and monosymptomatic dysphasic status epilepticus. Neurology 26: 244, 1976.

8) Hamilton NG, Matthews T. Aphasia. The sole manifestation of focal status epilepticus. Neurology 29: 745, 1979.

9) Hojo K, Fukushima Y. A case of partial status epilepticus with various neurophysiological symptoms. Folia Psychiatr Neurol Jpn 33: 291, 1979.

10) Reiley TL, Massey EW. The syndrome of aphasia, headaches, and left temporal spikes. Headache 20: 90, 1980.

11) Racy A, Osborn MA, Vern BA, Molinari GF. Epileptic aphasia. First onset of prolonged monosymptomatic status epilepticus in adults. Arch Neurol 37: 419, 1989.

12) Dinner DS, Lueders H, Lederman R, Gretter TE. Aphasic status epilepticus. A case report. Neurology 31: 888, 1981.

13) Marrosu F, Brundu A, Rachele MG, Marrosu G. Epileptic aphasia as dynamic disturbance. A case report. Acta Neurol (Napoli) 5: 43, 1983.

14) Knight RT, Cooper J. Status epilepticus manifesting as reversible Wernicke's aphasia. Epilepsia 27: 301, 1986.

15) Rosenbaum DH, Siegel M, Barr WB, Rowan AJ. Epileptic aphasia. Neurology 36: 822, 1986.

16) Primavera A, Bo G-P, Venturi S. Aphasic status epilepticus. Eur Neurol 28: $255,1988$.

17) Thomas P, Kullmann B, Chatel M. Aphasia as sole manifestation of status epilepticus.. Rev Neurol (Paris) 147: 246, 1991.

18) Suzuki I, Shimizu H, Ishijima B, Tani K, Sugishita M, Adachi N. Aphasic seizure caused by focal epilepsy in the left fusiform gyrus. Neurology 42: 2207, 1992.

19) Wells CR, Labar DR, Solomon GE. Aphasia as the sole manifestation of simple partial status epilepticus. Epilepsia 33: 84, 1992. 